V.N., Siarkowski, M., Sylwester, B., and sylwester, J.: 1983, Acta Astronomica 33, 431-460.

Stenflo, J.O.: 1982, Solar Phys. 80, 209-226.

Stenflo, J.O.: 1984a, in Proc. Internat. Workshop on Solar Physics and Interplanetary Travelling Phenomena, Kunming, China (in press).

Stenflo, J.0.: 1984b, in Solar Maximum Analysis Symp. 3, XXV COSPAR, Graz, Adv. Space Res. (in press).

Stenflo, J.O.: 1984C, in M.J. Hagyard (ed.), MSFC Workshop on Measurements of Solar Vector Magnetic Fields, NASA CP (in press).

Stenflo, J.O., Harvey, J.W., Brault, J.W., and Solanki, S.: 1984, Astron. Astrophys. 131, 333-346.

Strong, K.T., Alissandrakis, G.E., and Kundu, M.R.: 1984, Astrophys. J. 277, 865873.

Tang, F.: 1983, Solar Phys. 89, 43-50.

Tang, F. et al.: 1983, Adv. Space Res. 2, 65.

Urpo, S. \& 5 others:1982, Phys. Solariterr., Potsdam 19, 5.

Wagner, W.J. and Wagner, J.J.: 1984, Astron. Astrophys. 133, 288-292.

Wang, S.-G. et al.: 1984, in Proc. Internat. Workshop on Solar Physics and Interplanetary Travelling Phenomena, Kunming, China (in press).

Webb, D.F., Davis, J.Mi, Kundu, M.R., and Velusamy, T.: 1983, Solar Phys. 85, 267-283.

Willson, R.C.: 1984, Space Science Rev. 38, 203-242.

Wilson, P.R. and Simon, G.W.: 1983, Astrophys. J. 273, 805-821.

Wu, S.T., Hu, Y.Q., Krall, K.R., Hagyard, M.J., and Smith, J.B., Jr.: 1984, Solar Phys. 90, 117-131.

Zirin, H.: 1983, Astrophys. J. 274, 900-909.

Zirin, H.: 1984, Astrophys. J. 281, 884-885.

Zwaan, C. et al.: 1985, Solar Phys. 95, 3-14.

\title{
VIII. Theory of Flares
}

$$
\text { (E.R. Priest) }
$$

Magnetohydrodynamic (MHD) theory for the initiation and development of solar flares has developed considerably over the past 3 years and represents one of the liveliest areas of solar physics (Hood \& Priest, 1981a, Priest 1983a,b, Schindler 1982, Van Hoven 1982, Syrovatskil et al. 1983). This has been stimulated by a thorough analysis of the skylab observations and also by the startling new observations from the Solar Maximum Mission (SMM). In addition, the realization that flares appear to form two basic types, namely, small simple-loop flares and large two-ribbon flares, has focussed the imagination of theorists (e.g., Priest 1981, 1982), even though reality may be somewhat more complex. In the former type, a single-loop structure brightens up and decays without moving; whereas in the latter, an active region filament erupts and then two ribbons of chromospheric emission form and separate, with an arcade of hot and cool loops joining them.

The basic theory for hydrodynamic flow in a rigid loop and for magnetic field reconnection has been studied in depth, as summarized below. Major theoretical problems have been to try and understand how the magnetic field can become unstable and so initiate a flare in the two basic geometries, namely, a loop and an arcade. Also, the creation of post-flare loops by magnetic reconnection as the magnetic field closes back down in the main phase of a two-ribbon event has been modeled, and the roles of emerging flux are being clarified.

All these are at present active topics and one expects much theoretical progress over the next few years. In particular, the coupling of loop flow to the magnetic field should be studied, and the details of the new fast reconnection regions and of the nonlinear development of tearing should be worked out. Coupling the MHD to the various mechanisms for particle acceleration, such 
as turbulence and shock waves, is ready to be attempted, and the details of the flux emergence need to be studied. Also, a study of the nonlinear development of the MHD instabilities thought to initiate a flare has hardly begun.

\section{A. LOOP HYDRODYNAMICS}

Many authors have been tackling the hydrodynamic problem of the thermal response of plasma in a rigid magnetic tube to a sudden deposition of heat due to, for example, particle beams or magnetic reconnection. The fluid equations of mass continuity, momentum, and energy for one-dimensional flow in a loop of given shape are solved numerically for a variety of heat sources and loop shapes and lengths, representing an extension of the earlier pioneering work on chromospheric evaporation by Antiochos and sturrock (1982) and somov et al. (1977). After an additional heat source is suddenly switched on, pairs of conduction fronts and shock waves propagate down both legs of the loop away from the site of the source. When they reach the loop footpoints, the chromosphere is heated and expands upward (i.e., evaporates) to fill the loop with hot dense plasma. After some time, this plasma cools by radiation and conduction and then drains back down. Superimposed on this process, one finds oscillations with a period equal to the time it takes to propagate along the loop. [For reviews, see Craig (1981), Somov \& Spektor (1982), and Pallavicini et al. (1983).] Recent work in numerical simulation can be classified into two main groups. One group involves the hydrodynamic response to the injection of an electron beam (Somov et al. 1981, Nagai \& Emslie 1984). The other group comprises conduction-heated models (Wu et al. 1981, Smith \& Harmony 1982, Pallavicini \& Peres 1983, Nagai et al. 1983, Cheng et al. 1983, Serio 1982). Peres et al. (1982) have modeled the optically thick footpoints and find that chromospheric heating is much more efficient at evaporating material than coronal heating. With a Lagrangian formalism, Craig et al. (1982) show how a summit heat pulse raises the summit temperature and later (by evaporation) the summit density, after which both decline as the plasma drains back down. Antiochos \& Sturrock (1982) suggest that supersonic downflows in the late phase could be driven by footpoint cooling. MacNeice et al. (1984) and Pallavicini et al. (1983) have predicted the spectroscopic consequences of heating and evaporation (see also Doschek et al. 1982, 1983). In a series of papers, McClymont \& Canfield (1983a,b) and collaborators (Canfield et al. 1983, An et al. 1983, Ricchiazzi \& Canfield 1983) have investigated flare-loop radiative hydrodynamics with the aim of examining the role of various energy-transport mechanisms in a flaring loop.

\section{B. MAGNETIC RECONNECTION}

Our basic understanding of reconnection has been changed recently by some numerical experiments, which have linked the two previous strands of theory, namely, tearing mode instability and fast petschek-Sonnerup reconnection, and have presented us with new surprises (e.g., Vasyluinas 1983, Heyvaerts 1983, Priest 1985).

\section{Tearing Mode Instability}

The theory for this reconnection instability in a current sheet or a sheared magnetic field has been recently extended. The effect of photospheric line tying may be to stabilize a loop (Mok \& Van Hoven 1982) or an arcade (Migliuolo \& Cargill 1983). An important development is the discovery of a radiative tearing mode, which is typically a hundred times faster than normal tearing for coronal parameters (Van Hoven et al. 1983, Tachi et al. 1983, Van Hoven \& steinolfson 1983, Steinolfson \& Van Hoven, 1982, 1984). The temperature dependence of the magnetic diffusivity couples the induction equation to an energy equation containing joule heating, thexmal conduction, and optically thin radiative losses. This modifies considerably the normal tearing and radiative modes. Van Hoven (1981) has discussed the energetics of tearing. The tearing mode may develop nonlinearly along several pathways, depending on the geometry and the 
parameter regime. At large values of the Lundquist number, Steinolfson \& Van Hoven (1983a) find Rutherford saturation when the wavelength is twice the shear length, but a much faster growth at larger wavelengths. In a flux tube, the coupling of modes on different surfaces can be important. Also, if several islands form, they may rapidly combine by the coalescence instability (Bhattacharjee et al. 1983, Biskamp 1982). When the boundary conditions are free enough Forbes \& Priest (1982a, 1983a) have shown that it is possible for tearing to develop nonlinearly into the fast steady state of Petschek-Sonnerup reconnection. Steinolfson \& Van Hoven (1983b) have followed the nonlinear development of tearing at Lundquist numbers of $10^{\circ}$. They find a considerably slowing of the growth, with 808 of the magnetic energy going into thermal energy and a maximum electric field of only one-thousandth of the Dreicer field. Also, at long wavelengths, secondary vortices can create a new magnetic island at the $x$ points.

\section{Fast Reconnection}

The second main theme of reconnection theory has been the fast nonlinear state of Petschek-Sonnerup reconnection, which may either develop from linear tearing or be driven by pushing separate flux systems together. The current sheet bifurcates into two pairs of slow shock waves, which stand in the flow and convert inflowing magnetic energy into the heat and kinetic energy of hot fast jets. A rigorous compressible treatment of the Petschek extreme has now been completed (Soward \& Priest 1982). In general the external boundary conditions at the sources of inflowing plasma produce a hybrid Petschek-sonnerup regime. However, when reconnection develops locally from the tearing mode, the nonlinear state tends to be Petschek-like (Forbes \& Priest 1982a), whereas when the reconnection is ariven from outside, the nonlinear state can be closer to the pure sonnerup regime (Forbes 1984). The steady Petschek-Sonnerup mode is possible when the inflow speed ( $v$ ) of plasma at large distances is less than a maximum speed $\left(v_{\text {max }}\right)$, whose value depends on the magnetic Reynolds number and external boundary conditions. For pure Petschek reconnection, it is typically $0.01 v_{A}$, but for pure sonnerup reconnection, it is roughly the Alfvén speed ( $v_{A}$ ) at large distances. A new discovery is that fast-mode shocks may be present in the outflowing hot jets (Forbes \& Priest 1983a). These have the effect of degrading the kinetic energy to heat and may be very efficient at accelerating particles. Recent numerical experiments (Biskamp 1982, Forbes \& Priest 1982a, $1983 a, b)$ have revealed two new regimes of fast unsteady reconnection when the Petschek-Sonnerup mechanism breaks down. The flux pile-up regime occurs when the inflow of plasma is faster than $v_{\max }$, such as when reconnection is driven by an ideal instability. The impulsive bursty regime occurs when the central current sheet goes unstable to secondary tearing and produces a more.rapid energy release in a series of bursts as the islands coalesce.

\section{THE FLARE INSTABILITY}

\section{Kink Instability of Loop}

The preflare magnetic configuration has been modeled by a single loop, and its magnetic stability has been investigated, both for simple-loop and two-ribbon flares. Usually, the loop curvature has been neglected, but recently the important stabilizing effect of photospheric line tying has been included. It keeps the loop stable until the amount of twist in the loop exceeds a critical value, typically between $2 \pi$ and $4 \pi$, depending on the particular equilibrium, the plasma beta, and the details of the line tying. The most complete analysis has been presented by Hood \& Priest (1981b) and Einaudi \& Van Hoven (1983), building on earlier work (e.g., Van Hoven et al. 1981, Van Hoven 1981, Illing et al. 1981, song \& Cao 1983a,b). Recently, An (1983) investigated the radiative modes in a loop and found them unstable for small twist. 


\section{Eruptive Instability of Arcade}

For two-ribbon flares the preflare magnetic configuration has recently been modeled more accurately by a force-free cononal arcade, and its stability has been tested (including photospheric line tying) by Birn \& Schindler (1981), Schindlex et al. (1983), Ray \& Van Hoven (1982), Hood (1983a), and Migliuolo \& Cargill (1983). The original analysis of Hood \& Priest (1980) found that a simple arcade with its magnetic axis below the photosphere tends to be unstable. However, arcades with their magnetic axis above the photosphere are configurations within which an active region (or plage) filament may well form. Such filaments invariable erupt just before a two-ribbon event, slowly at first and then much more rapidly at flare onset. This type of coronal arcade has been found by Hood \& Priest (1981b) to become unstable when either the height of the magetic axis (and therefore the filament) or the amount of shear become too great. This suggests that the eruption of the arcade may be caused by a spontaneous eruptive instability when the filament height or magnetic shear become too great. Several equilibrium models for magnetostatic arcades with a balance between the Loxentz force, a pressure gradient, and gravity have been set up by Heyvearts et al. (1982), Zweibel \& Hundhausen (1982), and Melville et al. (1983). Using the energy method, schindler et al. (1983) have obtained a sufficient condition for stability of such fields. When there is no axial field, the conditions also becomes necessary, but Migliuolo et al. (1984) have shown that interchange modes with very short wavelengths along the arcade are unstable, which may be important for generating small-scale structure. In addition, the arcade becomes unstable when the plasma pressure gradient is too large. Zweibel (1982) has derived a sufficient condition for stability and for one paxticular configuration has shown a tendancy for instability when a magnetic island is present. Hood (1983b) has extended her analysis and investigated the instability threshold in more detail by including the effect of magnetic tension, which tends to make the field stable for small plasma pressure. Flares are often accompanied by a coronal transient, for which a theory has been developed by Low (1983) based on a simple self-similar model. Numerical simulations have been presented by Steinolfson (1982), and the et al. (1983).

\section{CREATION OF FLARE LOOPS}

During the preflare phase of a two-ribbon flare, the filament embedded in a magnetic arcade rises slowly, possibly due to the above eruptive instability. The onset of the flare itself coincides with the start of the much more rapid exuption of the filament, which probably occurs because the magnetic field lines of the stretched-out arcade start to reconnect below the filament (Priest 1981). During the main phase, the reconnection continues and creates hot postflare loops with $H \alpha$ ribbons at their footpoints as the field closes down. The source of the immense mass of plasma that is subsequently observed to be falling down along cool post-flare loops is an upflow of plasma from the chromosphere along the open fleld lines before they reconnect. The cause of the upflow may well be evaporation driven by thermal conduction or by fast particles that are accelerated at the shocks associated with the reconnection process (Forbes \& Priest 1983b). In the original Kopp-Pneuman model, the plasma was heated by gas dynamic shocks to only a few million degrees. Instead, Cargill \& Priest (1982, 1983) have suggested that the shocks should be slow magnetoacoustic in character, which can release extra energy from the magnetic field and can heat the upflowing plasma to the temperature of $20 \mathrm{million}$ degrees or. more observed in hot loops. Forbes has conducted a numerical experiment on the line-tied reconnection thought to be occurring below the erupting filament (Forbes \& Priest 1982a, 1983a,b). Open field lines that are stretched out go unstable to tearing, which develops nonlinearly into a quasi-steady Petschek regime with a fast shock in the downflowing jet. The field lines close down, with the $x$ type neutral point rising and a plasmoid being ejected upward. In the subsequent development, the 
sheet thins and the Petschek mode goes unstable, being transformed into the impulsive bursty regime. Secondary tearing and coalescence creates and annihilates neutral point pairs repetitively, with fast energy release in an impulsive manner. Forbes has also set up a simple kinematic model which describes the upward motion of the neutral line and the underlying hot loops in the main phase of a two-ribbon flare (Forbes \& Priest 1982b).

\section{E. ROLE OF EMERGING FLUX AND SATELLITE SUNSPOTS}

Small regions of emerging flux or satellite sunspots are often observed before flares, signifying the interaction of separate magnetic flux systems by either vertical or horizontal motions and the creation of current sheets at the interface between the two flux systems. The first role of such flux evolution is to create small flares; it has been estimated by solving the energy balance within the sheet (Milne \& Priest 1981). The second role is to build up magnetic energy in the coronal reservoir by increasing the complexity and shear in the active region. The third role is to trigger large flares by initiating energy release in the more extensive overlying field (e.g., Hood \& Priest 1981c). In particular, it may push up the filament or tear away some of the overlying field lines, or initiate large-scale reconnection (Priest 1984). Forbes has conducted a numerical experiment on flux emergence and finds that the whole process can be much more complicated and dynamic than previously thought (Forbes 1984). The flux emerges at a high speed and reconnects at first in a flux pile-up regime. Later, the flux pinches off and forms a plasmoid. In the future, it would be interesting to see whether experiments at magnetic Reynolds numbers higher than 2000 would give a more turbulent reconnection state. There has been a continuation of the earlier work on the behavior of the current sheet at the interface between new and old flux. Forbes et al. (1982) considered the evolution of the sheet in response to an enhanced turbulent resistivity. Sakai \& Washimi (1981, 1982) suggested that fast magnetoacoustic waves generated by the emerging flux may arive tearing with a faster growth than normal. The state of the high temperature turbulent current sheet approprlate to the main phase has been studied by Somov and co-workers (Somov 1981, 1983, Somov \& Titov 1983, Somov et al. 1982). They include an anomalous conductivity and heat flux as well as the effect of transverse and longitudinal field components, and demonstrate that the sheet liberates enough power for all phases of the flare. An improved model for the transverse structure of a current sheet has been established by Milne \& Priest (1981) who deduce the way that the critical sheet height for flare triggering depends on magnetic field strength and emergence speed.

\section{References}

An, C. - H.: 1983, Astrophys. J. 264(1), 302-308.

An, C.-H., Canfield, R. C., Fisher, G. H., and McClymont, A.N.: 1983, Astrophys. J. $267(1), 421-432$.

Antiochos, S.K. and Sturrock, P.A.: 1982, Astrophys. J. 254, 343-348.

Bhattacharjee, A., Brunel, F., and Tajima, T.: 1983, Phys. Fluids 26(11), 33323337.

Birn, J. and Schindler, K.: 1981, in E.R. Priest (ed.), Solar Flare Magnetohydrodynamics, Gordan and Breach, Iondon, Chapter 6.

Biskamp, D.: 1982, Phys. Letters 87A(7), 357-360.

Canfield, R.C., Fisher, G.H., and McClymont, A.N.: 1983, Astrophys. J. 265(1), 507-518.

Cargill, P.J. and Priest, E. R.: 1982, Solar Phys. 76, 357-375.

Cargill, P.J. and Priest, E.R.: 1983, Astrophys. J. 266(1), 383-389.

Cheng, C.-C., Oran, E.S., Doschek, G.A., Boris, J.P., and Mariska, J.T.: 1983, Astrophys. J. 265(2), 1090-1102.

Craig, I.J.D.: 1981, in E.R. Priest (ed.), solar Flare Magnetohydrodynamics, Gordan and Breach, pp. 217-336.

Craig, I.J.D., Robb, T.D., and Rollo, M.D.: 1982, Solar Phys. 76, 331-355. 
Doschek, G.A., Boris, J.P., Cheng, C.-C., Mariska, J.T., and Oran, E.S.: 1982, Astrophys. J. 258, 373-383.

Doschek, G.A., Cheng, C.-C., Oran, E.S., Boris, J.P., and Mariska, J.T.: 1983, Astrophys. J. 265(2), 1103-1119.

Einaudi, G. and Van Hoven, G.: 1983, Solar Phys. 88(1/2), 163-177.

Forbes, T.G.: 1984, in Proc. Solar Maximum Analysis Symp., Graz (in press).

Forbes, T.G. and Priest, E.R.: 1982a, Solar Phys. 81, 303-324.

Forbes, T.G. and Priest, E.R.: 1982b, Planet. Space Sci. 30, 1183-1197.

Forbes, T.G. and Priest, E.R.: 1983a, Solar Phys. 84(1/2), 169-188.

Forbes, T.G. and Priest, E.R.: 1983b, Solar Phys. 88(1/2), 211-218.

Forbes, T.G., Priest, E.R., and Hood, A.W.: 1982, J. Plasma Phys. 27(1), $157-176$.

Heyvaerts, J.: 1983, Adv. Space Res. 2(9), 21-26.

Heyvaerts, J., Lasry, J.M., Schatzman, M., and Witomsky, P.: 1982, Astron. Astrophys. 111, 104-112.

Hood, A.W.: 1983a, Solar Phys. 87(2), 279-299.

Hood, A.W.: 1983b, Solar Phys. 89(2), 235-242.

Hood, A.W. and Priest, E.R.: 1980, Solar Phys. 66, 113-134.

Hood, A.W. and Priest, E.R.: 1981a, in R.M. Bonnet and A.K. Dupree (eds.), NATO Advanced Study Institutes Series C, Vol. 68, D. Reidel Publ. Co., Dordrecht, pp. 509-531.

Hood, A.W. and Priest, E.R.: 1981b, Geophys. Astrophys. Fluid Dyn. 17, 297-318.

Hood, A.W. and Priest, E.R.: 1981c, Solar Phys. 73, 289-311.

Illing, R.M.E., House, L.L., Wagner, W.J., and Sawyer, C.: 1981, Bull. AAS, 13, 862 .

Low, B.C.: 1983, in J.O. Stenflo (ed.), IAU Symp. No. 102, D. Reidel Publ. Co., Dordrecht, pp. 467-471.

MacNeice, P., McWhirter, R.W.P., Spicer, D.S., and Burgess, A.: 1984, Solar Phys $90(2), 357-382$.

McClymont, A.N. and Canfield, R.C.: 1983a, Astrophys. J. 265(1), 483-496.

McClymont, A.N. and Canfield, R.C.: 1983b, Astrophys. J. 265(1), 497-506.

Melville, J.P., Hood, A.W., and Priest, E.R.: 1983, Solar Phys. 87(2), $301-307$.

Migliuolo, S. and Cargill, P.G.: 1983, Astrophys. J. $271(2), 820-831$.

Migliuolo, S., Cargill, P.G., and Hood, A.W.: 1984, Astrophys. J. 281, 413-418.

Milne, A.M. and Priest, E.R.: 1981, Solar Phys. 73, 157-181.

Mok, Y. and Van Hoven, G.: 1982, Phys. Fluids 25, 636.

Nagai, F. and Emslie, A.G.: 1984, Astrophys. J. 279, 896-908.

Naga1, F., Wu, S.-T., and Tandberg-Hanssen, E.: 1983, Solar Phys. 84(1/2), 271283.

Pallavicini, R. and Peres, G.: 1983, Solar Phys. 86(1/2), 147-157.

Pallavicini, R., Peres, G., Serio, S., Vaiana, G., Acton, L., Leibacher, J., and Rosner, R.: 1983, Astrophys. J. 270(1), 270-287.

Peres, G., Rosner, R., Serio, S., and Vaiana, G.S.: 1982, Astrophys. J. 252, 791799.

Priest, E.R. (ed.): 1981, Solar Flare Magnetohydrodynamics, Gordan and Breach, London.

Priest, E. R.: 1982, Fundam. Cosmic Phys. 7(4), 363-445.

Priest, E.R.: 1983a, in Activity in Red Dwarf stars, IAU Colloq. 71, pp. 545-558.

Priest, E. R.: 1983b, Solar Phys. 86(1/2), 33-45.

Priest, E.R.: 1984, in Proc. Solar Maximum Analysis Symp., Graz (in press).

Priest, E. R.: 1985, Rep. Prog. Phys. (in press).

Ray, A. and Van Hoven, G.: 1982, Solar Phys. 79, 353-364.

Ricchiazzi, P.J. and Canfield, R.C.: 1983, Astrophys. J. 272(2), 739-755.

Saka1, J.-I. and Washimi, H.: 1981, Report IPPJ-540, Nagoya University, Japan.

Sakai, J.-I. and Washimi, H.: 1982, Astrophys. J. 258, 823-824.

Schindler, K.: 1982, Phys. Scripta T2(1), 163-168.

Schindlex, K., Birn, J., and Janicke, L.: 1983, Solar Phys. 87(1), 103-133.

Serio, S.: 1982, Mem. Soc. Astron. Ital. 53(4), 865-870.

Smith, D.F. and Harmony, D.W.: 1982, Astrophys. J. 252, 800-809.

Somov, B.V.: 1981, Fiz. Inst. AN SSSR. Preprint 15. 
Somov, B.V.: 1983, Zemlya Vselennaya 6, 13-17.

Somov, B.V. and Spektor, A.R.: 1982, Space Sci. Rev. 32, 27-41.

Somov, B.V. and Titov, V.S.: 1983, Pisma Astron Zh. 9(1), 48-52 (in Russian).

Somov, B.V., Spektor, A.R., and Syrovatskil, S.I.: 1977, Izv, AN SSSR. Ser. Fiz 41, 273-287.

Somov, B.V., Syrovatskil, S.I., and Spektor, A. R.: 1981, Solar Phys. 73, $145-155$.

Somov, B.V., Stepanov, V.E., Stepanyan, N.N., and Tomozov, V.M.: 1982, Issledovaniya po geomagnetizmu aeronomii i fizike Solntsa, Moskva 60, 112-131 (in Russian).

Song, M.-t, and Cao, T.-j.: 1983a, Acta Astron. Sin. 24(1), 1-9.

Song, M.-t. and Cao, T.-j.: 1983b, Chin. Astron. Astrophys. 7(3), 159-164

Soward, A.M. and Priest, E.R.: 1982, J. Plasma Phys. 28(2), 335-367.

Steinolfson, R.S.: 1982, Astron. Astrophys. 115, 39-49.

Steinolfson, R.S. and Van Hoven, G.: 1982, Phys. Fluids 26, 2590-2602.

Steinolfson, R.S. and Van Hoven, G.: 1983a, Phys. Fluids 26(1), 117-123.

Steinolfson, R.S. and Van Hoven, G.: 1983b, Bull. AAS 15(2), 710.

Steinolfson, R.S. and Van Hoven, G.: 1984, Astrophys. J. $276,391$.

Syrovatskil, S.I., Bulanov, S.V., and Dogel', V.A.: 1983, Astrophys. Space Phys. Rev. 2, 385.

Tachi, T., Steinolfson, R.S., and Van Hoven, G.: 1983, Phys. Fluids 26(10), 29762982.

Van Hoven, G.: 1981, in E.R. Priest (ed.), Solar Flare Magnetohydrodymamics, Gordon and Breach, Iondon, Chapter 4.

Van Hoven, G.: 1982, Mem. Soc. Astron. Ital. 53(2), 441-460.

Van Hoven, G. and Steinolfson, R.S.: 1983, Bull. AAS 15(2), 700 .

Van Hoven, G., Ma, S.S., and Einaudi, G.: 1981, Astron. Astrophys. 97, $232-234$.

Van Hoven, G., Steinolfson, R.S., and Tachi, T.: 1983, Astrophys. J. 268(2), 860864.

Vasyliunas, V.M.: 1983, in R.L. Carovillano and J.M. Forbes (eds.), Astrophysics and Space Science Library, Vol. 104, D. Reidel Publ. Co.. Dordrecht, pp. 255260 .

Wu, S.-T., Kan, L.C., Nakagawa, Y., and Tandberg-Hanssen, E.: 1981, Solar Phys. $70,137-148$.

Wu, S.-T., Wang, S., Dryer, M., Poland, A.I., sime, D.G., wolfson, C.J., Orwig, L.E., and Maxwell, A.: 1983, Solar Phys. 85(2), 351-373.

Zweibel, E.G.: 1982, Astrophys. J. Letters 258, L53-L56.

Zweibel, E.G. and Hundhausen, A.J.: 1982, Solar Phys. 76, 261-299.

E. Tandberg-Hanssen

President of Commission 